\title{
Multi-parametric characterization of brain-wide hemodynamic and calcium responses to sensory stimulation in mice
}

\author{
Zhenyue Chen ${ }^{1,2}$, Quanyu Zhou ${ }^{1,2}$, Xosé Luís Deán-Ben ${ }^{1,2}$, Irmak Gezginer ${ }^{1,2}$, Ruiqing Ni ${ }^{1,2}$, Michael \\ Reiss $^{1,2}$, Shy Shoham ${ }^{3, *}$, Daniel Razansky ${ }^{1,2, *}$ \\ ${ }^{1}$ Institute for Biomedical Engineering and Institute of Pharmacology and Toxicology, Faculty of Medicine, University of Zurich, \\ Switzerland \\ ${ }^{2}$ Institute for Biomedical Engineering, Department of Information Technology and Electrical Engineering, ETH Zurich, \\ Switzerland \\ ${ }^{3}$ Department of Ophthalmology and Tech4Health and Neuroscience Institutes, NYU Langone Health, New York, USA \\ *Correspondence to daniel.razansky@uzh.ch or shoham@nyu.edu
}

\begin{abstract}
Modern optical neuroimaging approaches are expanding our ability to elucidate complex brain function. Diverse imaging contrasts enable direct observation of neural activity with functional sensors along with the induced hemodynamic responses. To date, decoupling the complex interplay of neurovascular coupling and dynamical physiological states has remained challenging when employing single-modality functional neuroimaging tools. We devised a hybrid fluorescence optoacoustic tomography (FLOT) platform combined with a custom data processing pipeline based on statistical parametric mapping, accomplishing the first simultaneous noninvasive observation of both direct and indirect brain-wide activation patterns with optical contrast. Correlated changes in the oxy- and deoxygenated hemoglobin, total hemoglobin, oxygen saturation and rapid GCaMP6f fluorescence signals were observed in response to peripheral sensory stimulation. While the concurrent epifluorescence served to corroborate and complement the functional optoacoustic observations, the latter further aided in decoupling the rapid calcium responses from the slowly varying background in the fluorescence recordings mediated by hemodynamic changes. The hybrid imaging platform expands the capabilities of conventional neuroimaging methods to provide more comprehensive functional readings for studying neurovascular and neurometabolic coupling mechanisms and related diseases.
\end{abstract}

\section{Keywords}

Neuroimaging, brain imaging, functional optoacoustic tomography, epifluorescence, brain activation, hemodynamic response, GCaMP6f, electric paw stimulation

\section{Introduction}

Functional neuroimaging has become a primary tool in neuroscience and research into neurodegenerative diseases. Blood-oxygen-level-dependent (BOLD) functional magnetic resonance imaging ( $\mathrm{fMRI}$ ) is the mainstay for noninvasive mapping of brain function ${ }^{1}$. However, interpretation of activity-related BOLD signals is challenged by their dependence on multiple factors such as blood flow, blood volume, metabolic rate of oxygen and baseline physiological state ${ }^{2}$. Functional ultrasound imaging can provide additional information on the blood flow ${ }^{3}$. However, the blood flow changes similarly represent an indirect correlate of the underlying neural dynamics, which is insufficient for fully characterizing the complex interplay between neural activity and its accompanying hemodynamics. 
Optical imaging approaches arguably provide the most powerful means for retrieving functional and molecular information underlying brain activity. The emergence of GCaMP-type and other genetically encoded calcium indicators has facilitated fluorescence-mediated sensing of neural activation ${ }^{4}$, and has for example been widely employed to characterize resting state activity and stimulus-evoked responses in the mouse cortex ${ }^{5}$. In addition, hemoglobin exhibits distinct absorption spectra in its oxygenated ( $\left.\mathrm{HbO}\right)$ and deoxygenated $(\mathrm{HbR})$ states $^{6}$, which can be visualized with functional optoacoustic (fOA) tomography across 3D space and time, enabling the mapping of multiple hemodynamic parameters across the entire murine brain, not accessible with other modalities ${ }^{7-9}$. Imaging modalities based on optical contrasts can thus potentially massively advance our knowledge on the mechanisms of neurovascular coupling, that is, if the mapping of both the neuronal activity directly as well as its associated hemodynamic changes could be realized simultaneously. However, a detailed characterization and rigorous side-by-side validation of hemodynamic fOA readings against well-established, direct optical neuroimaging methods is currently lacking.

Here, we introduce a hybrid fluorescence and optoacoustic tomography (FLOT) platform for concurrent multi-parametric characterization of brain-wide hemodynamic and calcium responses to sensory stimulation in mice. A custom data processing pipeline inspired by statistical parametric mapping (SPM) was further developed to facilitate the functional data analysis. Simultaneous imaging of calcium and hemodynamic responses to electrical paw stimulation was performed in GCaMP6f mice, for which the GCaMP signals were shown to reflect changes in intracellular calcium corresponding to neural spiking activity $^{5,10}$. To the best of our knowledge, this represents the first successful observation of activation patterns (direct and indirect responses simultaneously) in the entire mouse cortex with optical contrast. The use of near-infrared (NIR) wavelengths in fOA imaging further enabled to reach deeper brain regions thus averting cross-talk between calcium and hemodynamic signals.

\section{Results}

\section{The hybrid fluorescence and optoacoustic tomography (FLOT) system}

The dedicated hybrid FLOT imaging system employs a flexible fiberscope for capturing fluorescence images in epi-illumination mode with $\sim 44 \mu \mathrm{m}$ pixel resolution. The distal end of the fiberscope was inserted into the central cavity of a spherical matric array transducer used for acquiring three dimensional (3D) fOA data with nearly isotropic spatial resolution which was estimated to be $113 \mu \mathrm{m}$ using microsphere measurements, slightly degrading at the periphery FOV (Fig. 1a and Fig. S1) ${ }^{11,12}$. Both modalities provided a $12 \times 12 \mathrm{~mm}$ field of view (FOV) along the lateral $x-y$ plane covering the entire mouse cortex. Trains of unipolar rectangular electrical pulses with $5 \mathrm{~ms}$ duration and $1.0 \mathrm{~mA}$ intensity were applied to the left hindpaw of Thy1-GCaMP6f mice. The trains consisted of 32 pulses with stimulus frequency of $4 \mathrm{~Hz}$ (i.e., $8 \mathrm{~s}$ total duration) repeated every $82 \mathrm{~s}$ (Fig. 1b). Five wavelengths (700, 730, 755, 800 and $850 \mathrm{~nm}$ ) were optimally selected for fOA to avoid significant spectral coloring while ensuring a small condition number of the spectral unmixing matrix (Fig. 1C). The unmixed hemodynamic components of $\mathrm{HbO}$ and $\mathrm{HbR}$ are shown in Fig. 1d. Subsequently, both fluorescence and fOA data were analyzed with a dedicated data processing pipeline (Fig. 1e). We employed regressors for analyzing the hemodynamic and calcium responses by convolving the stimulation pulse train with a hemodynamic response function (HRF) and a GCaMP calcium response function (CRF), respectively (see Methods for a detailed description). 

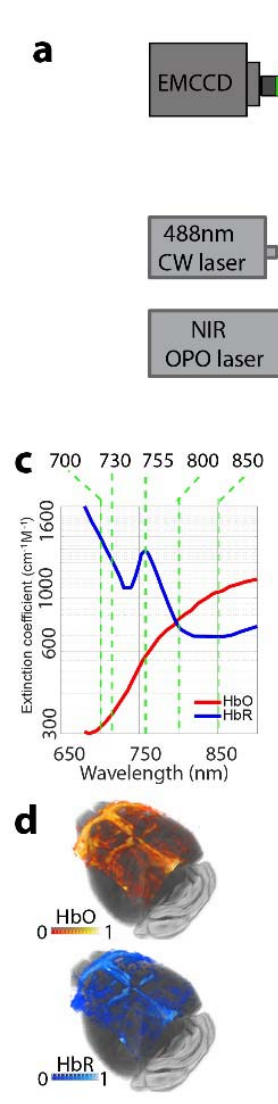

Fluorescence

Filters Fiberscope

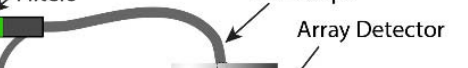

Th

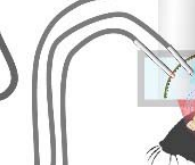

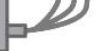

(1)

e
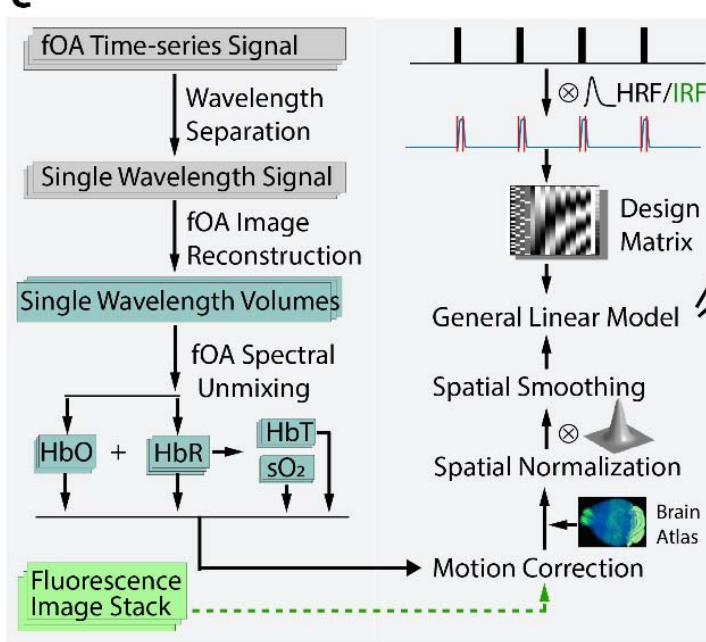
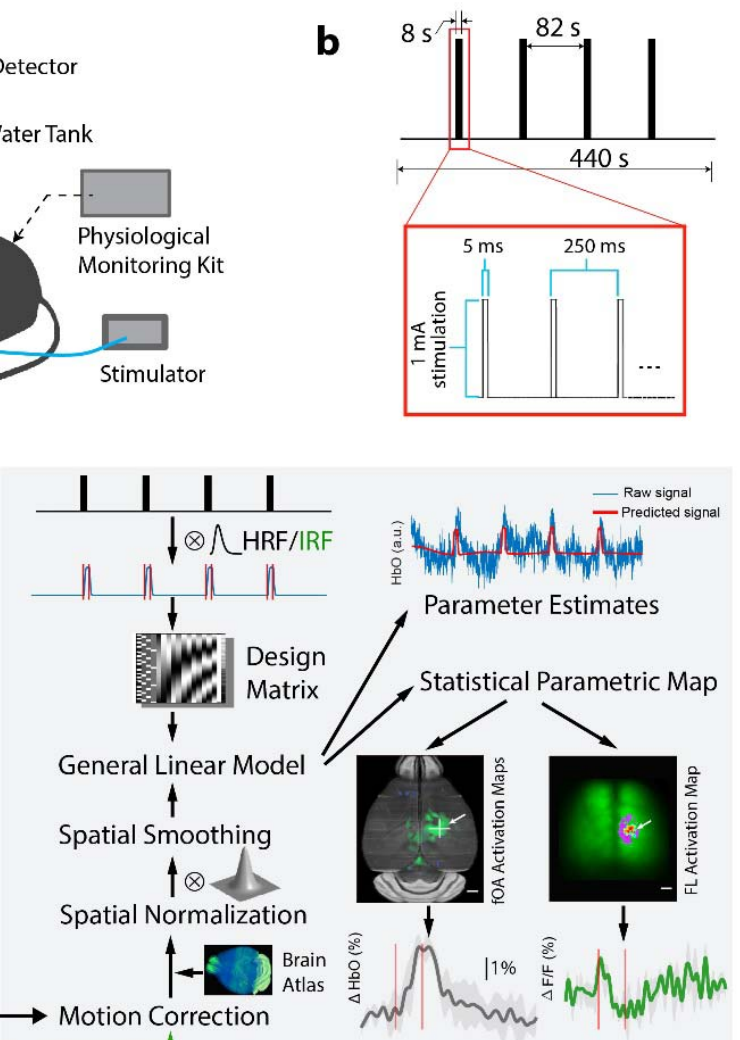

Figure 1 Layout of the hybrid FLOT platform for multi-parametric imaging of murine brain activation. a Schematics of the imaging system incorporating the simultaneous functional optoacoustic (fOA) and fluorescence readings. $\mathbf{b}$ Burst electric current stimulation paradigm applied to the left hindpaw. c Wavelength selection for the spectroscopic fOA imaging of blood oxygenation. The molar extinction spectra for $\mathrm{HbO}$ and $\mathrm{HbR}$ are plotted. d Spatial distribution of the spectrally unmixed $\mathrm{HbO}$ and $\mathrm{HbR}$ hemodynamic components in the brain. e The custom FLOT data processing pipeline includes fOA image reconstruction and spectral unmixing (left column), fluorescence data reshaping, pre-processing and the general linear model (middle column), and statistical parametric mapping (right column).

\section{GCaMP6f impulse Calcium Response Function (CRF)}

We first estimated the CRF of the rapid calcium activity using epifluorescence recordings acquired at a high frame rate of $200 \mathrm{~Hz}$ in Thy1-GCaMP6f mouse brains in vivo. The high frame-rate acquisitions were employed to extract well-defined response function profiles subsequently used in the fluorescence data processing pipeline. The scalp of Thy1-GCaMP6f mice was removed prior to imaging while keeping the skull intact (Fig. 2a), which resulted in clearly resolvable cortical vasculature. Temporal fluorescence signal profiles from two representative $0.3 \times 0.3 \mathrm{~mm}^{2}$ regions of interest (ROIs) were tracked in both the contralateral (opposite side from the stimulated paw) and ipsilateral hemispheres, as indicated in Fig. $2 a$. The recorded profiles were averaged across different stimulation bursts to reveal the activation time course (Fig. 2b). The averaged contralateral and ipsilateral signal profiles manifested a high degree of correlation, consisting of both spontaneous wave activity as well as sharp contralateral activity spikes evoked by the electric pulses. The respective averaged time-lapse images further reveal the spatiotemporal patterns of stimulus-evoked neuronal activation in the somatosensory cortex (Fig. 2c). In order 
to estimate the average stimulus-evoked fluorescence response (Fig. $2 \mathbf{d}$ and Fig. S2), we first applied a temporal notch filter with $0.2-3 \mathrm{~Hz}$ stop-band to the unaveraged time profiles in the selected contralateral ROI. Next, the filtered profiles were averaged for all the stimulation electric pulses with the resulting curve fitted to a gamma function $y=c t^{a-1} e^{-t / b} /\left[b^{a} * \Gamma(a)\right], \mathrm{a}=4.775, \mathrm{~b}=0.016, \mathrm{c}=0.079$. In the subsequent analysis, the gamma fitted curve was adopted as the characteristic GCaMP6f CRF to external electrical paw stimulation. The calculated correlation coefficient between the measured curve and the fitted gamma function is 0.991 with $p$-value $<10^{-5}$ for $t$ test. Other functional parameters were further calculated across all the stimulation trials, including the activation intensity $1.3 \pm 0.4 \%$, time-topeak (TTP) $0.063 \pm 0.013 \mathrm{~s}$, full-width-at-half-maximum (FWHM) of the calcium response $0.085 \pm 0.024 \mathrm{~s}$ and decay time $\left(T_{1 / 2}\right) 0.106 \pm 0.025 \mathrm{~s}$ (Fig. 2e). A linear regressor was then obtained by convolving the stimulation paradigm with the GCaMP6f CRF. Fluorescence data processing with the proposed pipeline rendered an activation map revealing the expected location of the activated region with high specificity (Fig. 2f). Compared to an activation map based on the difference image between activated frames and baseline recording, the proposed method exhibits superior robustness and has better localization specificity as it is less affected by the slow changes in light intensity affected by hemoglobin absorption variations (Fig. S3).

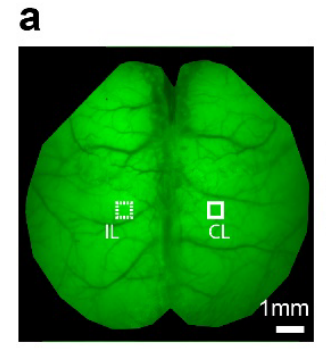

C

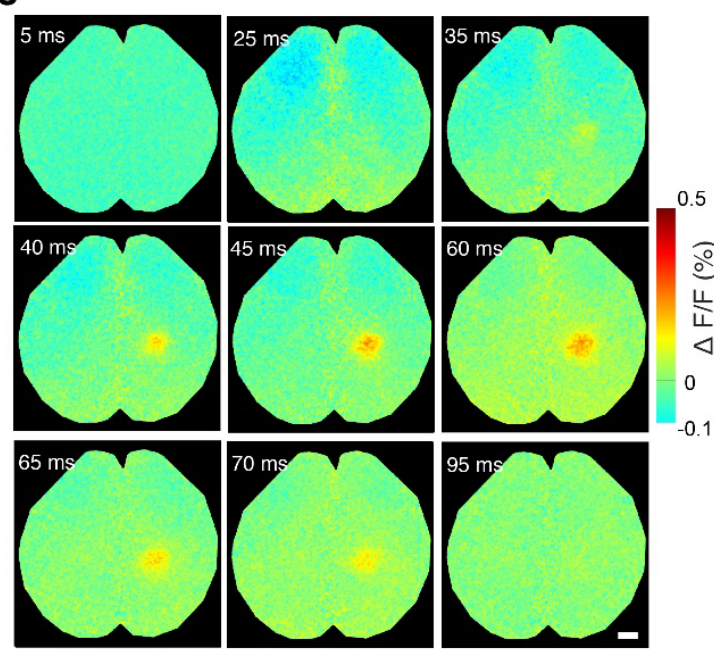

b

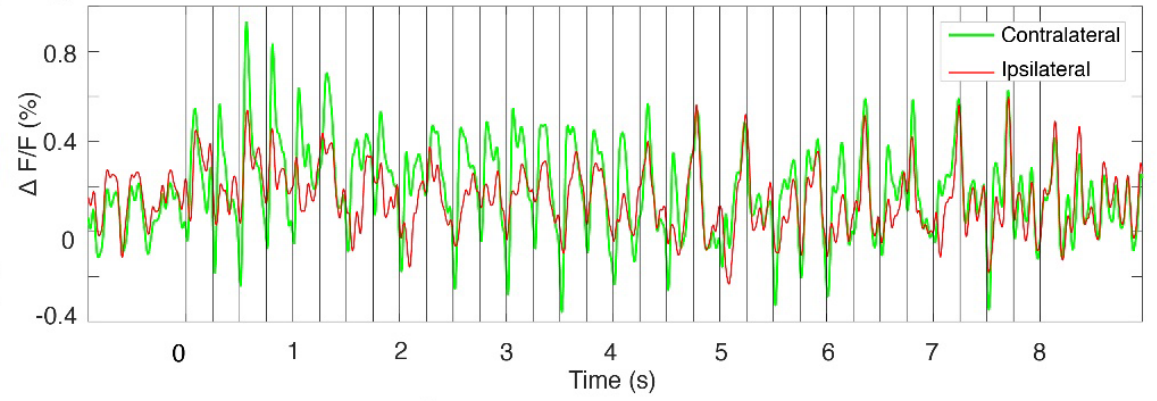

d

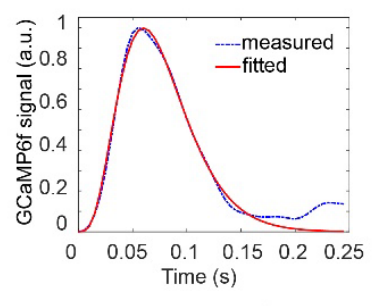

$\mathbf{f}$

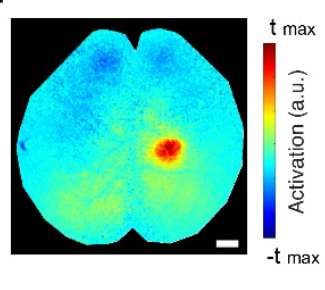

e

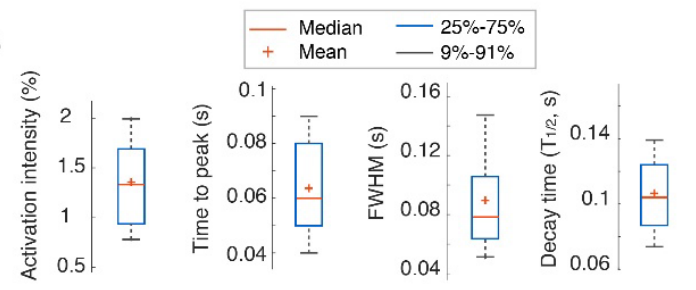

Figure 2 Assessment of the GCaMP6f impulse response function to electrical paw stimulation in mice. a Representative time-averaged epi-fluorescence image of the mouse brain. $\mathbf{b}$ Temporal fluorescence signal profiles spatially averaged over two $0.3 \times 0.3 \mathrm{~mm}^{2}$ sized ROIs in the contralateral (CL) and ipsilateral (IL) somatosensory cortex, as indicated in panel a. Each vertical line indicates single electric pulse. c Time-lapse images post the pulsed electric current stimulation obtained by averaging the responses from all the stimulation trials (pulses). $d$ Averaged 
stimulus-evoked response function estimated by averaging activation curves from 160 consecutive stimulation pulses fitted to a Gamma function. e Functional parameters extracted from all the stimulation trials. $f$ Activation map obtained from the proposed data processing pipeline. All scale bars: $1 \mathrm{~mm}$.

\section{FLOT reveals concurrent calcium and hemodynamic response maps}

Next, we simultaneously imaged calcium and hemodynamic responses in Thy1-GCaMP6f mice $(\mathrm{n}=6)$. Since the fOA imaging was performed in the near-infrared spectral window, the scalp was kept intact for 4 mice while it was removed for another 2 mice to mitigate image artifacts due to skin pigmentation (see Methods section). Activation maps corresponding to different hemodynamic components, namely $\mathrm{HbO}$, $\mathrm{HbR}$, total hemoglobin $(\mathrm{HbT})$ and oxygen saturation $\left(\mathrm{sO}_{2}\right)$, were further rendered from the fOA image sequences. Localized responses were clearly observed in the transverse, sagittal and coronal cross sections overlaid to the Allen P56 mouse brain atlas ${ }^{13}$ (Fig. 3a; voxels in the activation map are statistically significant with respect to a null hypothesis of no activation: $p<0.05$ one-sample t-test after false discovery rate (FDR) correction). Localized activity was detected within the primary somatosensory cortex as well as within the primary motor area on the contralateral side. No obvious activation was observed in the corresponding regions on the ipsilateral side. Since the HbR response was negatively correlated to the hemodynamic response regressor while other components were positively correlated, both positive and negative thresholds were applied to the calculated activation map (i.e., t-map) of each component. Multiple functional parameters were estimated from these fOA maps, i.e., the activation intensity, TTP, FWHM, decay time and activation volume size (Fig. 3b).

We next analyzed the concurrently-acquired fluorescence data. Due to the strong light scattering from the scalp, the fluorescence images appeared blurry compared to those acquired after scalp removal. Yet, localized brain activation upon electrical paw stimulation was clearly observed through the intact scalp. Moreover, increased light absorption by hemoglobin results in modulation of fluorescence signals by the hemodynamic responses in the activated region. A secondary hemodynamic activation map was thus obtained simultaneously from the fluorescence measurement by simply replacing the GCaMP CRF with the HRF in the construction of the regressor and overlaid onto the GCaMP activation map (Fig. 3c; both activation maps are thresholded at $70 \%$ of their corresponding maximum t-value).

Both unaveraged fluorescence and fOA signal time courses are shown in Fig. 3d, depicting the highly dynamic spontaneous fluctuations and stimulus evoked responses. Significant correlation exists between the simultaneously recorded fluorescence and $\mathrm{HbO} / \mathrm{HbR}$ responses, despite the fact that the signals were measured with two independent modalities, which may partially explain the slow background fluorescence signal fluctuations. These dynamics may further depend on the physiological status of the mouse, which is profoundly affected by many factors such as anesthesia and individual differences. Fractional averaged signal changes from representative ROls in contralateral hemisphere are shown in Fig. 3e. Interestingly, the lowpass filtered fluorescence signal peaked right after the paw stimulation due to the fast calcium-mediated response but then slowly declined below its baseline value gradually going back to the baseline, reflecting changes in light attenuation associated to increased local hemoglobin absorption in the activated regions.

Our results show that fOA provides diverse information on brain activation responses complementing the fluorescence readings. Further, it was observed that fractional hemodynamic signal changes significantly differ across various brain regions whereas fluorescence responses did not exhibit such spatial variability (Fig.S4). 
a
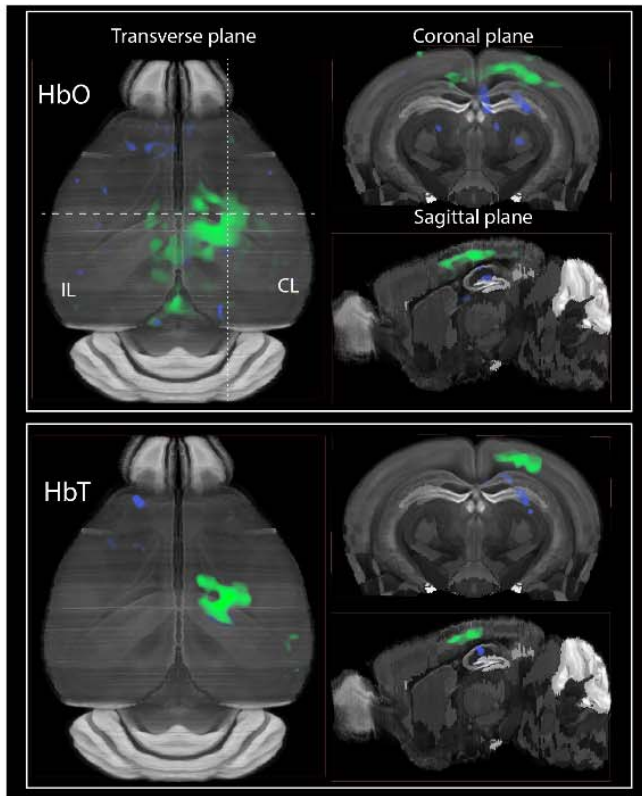

b

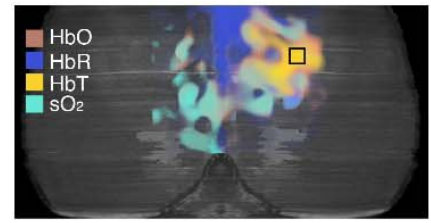

\begin{tabular}{c|l|l|l|l|l}
\hline $\begin{array}{l}\text { Hemodynamic } \\
\text { component }\end{array}$ & $\begin{array}{l}\text { Activation } \\
\text { intensity }(\%)\end{array}$ & TTP (\%) & FWHM (s) & $\begin{array}{l}\text { Decay time } \\
\left(\mathrm{T}_{1 / 2}, \mathrm{~s}\right)\end{array}$ & $\begin{array}{l}\text { Activation } \\
\text { volume }\left(\mathrm{mm}^{3}\right)\end{array}$ \\
\hline $\mathrm{HbO}$ & $1.8 \pm 0.7$ & $9.6 \pm 1.6$ & $7.4 \pm 1.6$ & $17.5 \pm 3.3$ & 0.77 \\
\hline $\mathrm{HbR}$ & $-1.1 \pm 0.4$ & $14.9 \pm 5.3$ & $10.9 \pm 5.8$ & $27.1 \pm 8.5$ & 0.60 \\
\hline $\mathrm{HbT}$ & $0.9 \pm 0.2$ & $11.2 \pm 7.6$ & $7.1 \pm 1.1$ & $13.3 \pm 0.8$ & 0.42 \\
\hline $\mathrm{sO}_{2}$ & $2.6 \pm 0.8$ & $10.0 \pm 1.5$ & $6.5 \pm 1.4$ & $16.0 \pm 0.4$ & 0.93 \\
\hline
\end{tabular}

d

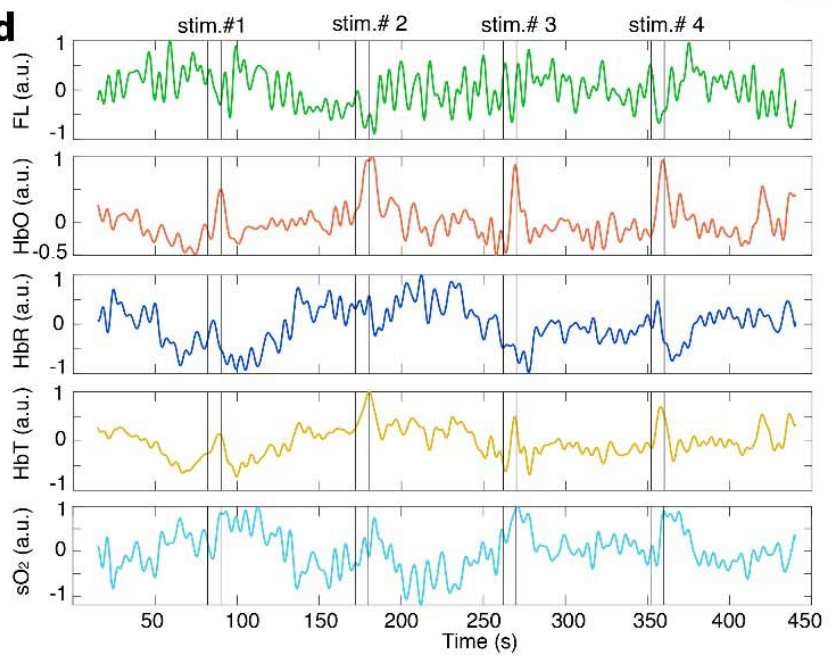

C
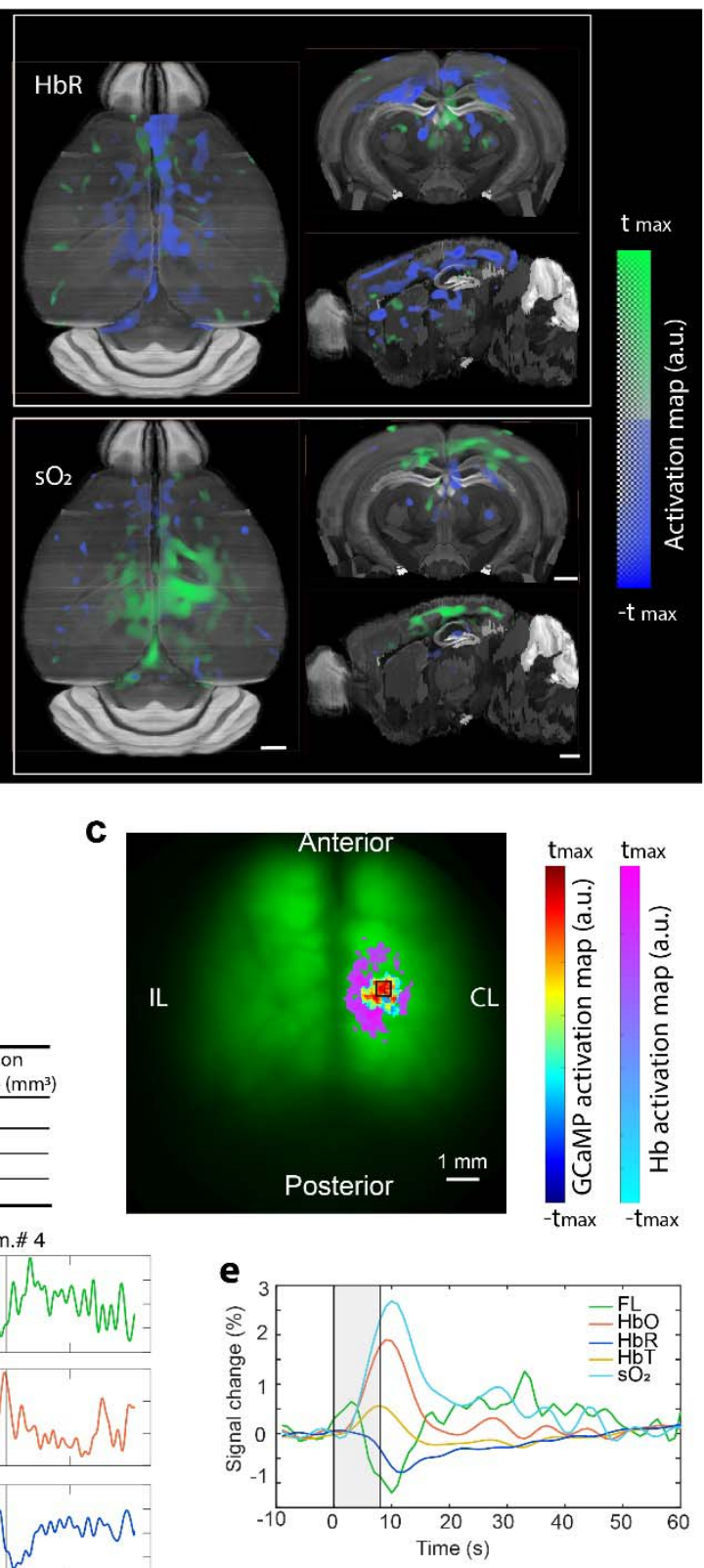

Figure 3 Concurrent measurement of calcium and hemodynamic responses in the mouse brain. a Transverse, sagittal and coronal views of the activation maps corresponding to $\mathrm{HbO}, \mathrm{HbR}, \mathrm{HbT}$ and $\mathrm{sO}_{2}$ components extracted from fOA images. $b$ Corresponding GCaMP and hemodynamic activation maps calculated from the fluorescence measurements overlaid with the fluorescence image of a GCaMP mouse brain with intact skull and scalp. c Superimposed fOA activation maps and statistics of the functional parameters from the ROI indicated by the black square situated at a depth of $\sim 300 \mu \mathrm{m}$ from the surface of the cortex. $\mathbf{d}$ Unaveraged time courses of the fluorescence and fOA signals from the ROIs indicated in $\mathbf{b}$ and $\mathbf{c}$. The fluorescence signal is lowpass filtered to 
emphasize slow trends (top row; Butterworth, cutoff frequency $0.5 \mathrm{~Hz}$ ). e Averaged activation time courses of fluorescence, $\mathrm{HbO}, \mathrm{HbR}, \mathrm{HbT}$ and $\mathrm{sO}_{2}$ from the $\mathrm{ROI}$ indicated in $\mathbf{b}$ and $\mathrm{c}$. $\mathrm{CL}$ : contralateral, $\mathrm{LL}$ : ipsilateral. All scale bars: $1 \mathrm{~mm}$.

\section{Multi-parametric analysis of stimulus-evoked brain activation}

Multi-parametric analysis was performed to characterize the coupling between simultaneous fluorescence and fOA readings. The same data processing pipeline was repeated for all mice $(n=6)$ across a total of 80 stimulation trials. Activation maps were first calculated using both the fluorescence and $\mathrm{fOA}$ images to infer activated brain regions and thereby to select ROIs for calculating the time courses. Spontaneous neural activity largely averages-out pre- and post-stimulation while the stimulation evoked responses are preserved (Fig. 4a). The fractional signal changes of the different hemodynamic components were then averaged across animals (Fig. $4 \mathbf{b}-\mathbf{e}, \mathrm{n}=6$ ). Strongly correlated signal increase in $\mathrm{HbO}$ (Fig. 4b), $\mathrm{HbT}$ (Fig. 4d) and $\mathrm{SO}_{2}$ (Fig. 4e) and $\mathrm{HbR}$ decline (Fig. 4c) were observed from the $\mathrm{fOA}$ measurements. As expected, these signal changes are closely correlated to the predicted hemodynamic response by convolving the HRF with the mean neural activity trace (green curve in Fig. 4a). Based on the fractional change in $\mathrm{HbO}$ and $\mathrm{HbR}$, the fluorescence signal contaminated by slowly varying changes in hemoglobin absorption was successfully corrected ${ }^{5}$ using estimates of the pathlength factors $x E x=0.56 \mathrm{~mm}^{-1}$ and $x E m=0.57 \mathrm{~mm}^{-1}$ (Fig. 4f).

The coupling between neural activity and the various hemodynamic response components was further studied by calculating the relationship between the peak values of the corresponding (simultaneously measured) single activation traces of different normalized response components (Figs. 4g-j). In Figs. 4g-h, the results show a strong coupling relationship between $\mathrm{HbT}$ and $\mathrm{sO}_{2}$ responses vs. both $\mathrm{HbR}$ and $\mathrm{HbO}$ responses, respectively. These results show that both $\mathrm{HbT}$ and $\mathrm{SO}_{2}$ responses saturate for high $\mathrm{HbO}$ responses, while they're uniformly linear across the full $\mathrm{HbR}$ response range. Further, by comparing the ranges and standard deviations between the different plots it is seen that both $\mathrm{HbT}$ and $\mathrm{sO}_{2}$ are more closely coupled with $\mathrm{HbO}$ than with $\mathrm{HbR}$. Besides, an overall stronger coupling of $\mathrm{sO}_{2}$ responses to both $\mathrm{HbO}$ and $\mathrm{HbR}$ was observed. The strong relationship between the different hemodynamic components extracted from the fOA measurement and the fluorescence signal corroborates the synergistic detection of neurovascular coupling phenomena in stimulus-evoked brain activation by the hybrid FLOT method.

Despite the strong correlation among fOA components, noticeable differences were observed in the calculated functional parameters (Fig. $4 \mathbf{k}$ ). $\mathrm{sO}_{2}(1.7 \pm 0.9 \%)$ presents stronger activation intensity as compared to $\mathrm{HbO}(1.6 \pm 0.9 \%), \mathrm{HbR}(-1.2 \pm 0.6 \%)$ and $\mathrm{HbT}(0.7 \pm 0.2 \%)$. In terms of TTP, the HbR readings $(15.7 \pm 5.9 \mathrm{~s})$ exhibit the most delayed response followed by $\mathrm{sO}_{2}(10.3 \pm 1.3 \mathrm{~s}), \mathrm{HbO}(10.3 \pm 1.4 \mathrm{~s})$ and $\mathrm{HbT}(9.9 \pm 2.9 \mathrm{~s})$. Similar pattern was observed in the FWHM values with $\mathrm{HbT}(11.5 \pm 4.9 \mathrm{~s})$ holding the longest response duration followed by $\mathrm{HbR}(11.3 \pm 6.5 \mathrm{~s}), \mathrm{HbO}(10.5 \pm 4.0 \mathrm{~s})$ and $\mathrm{sO}_{2}(10.3 \pm 3.8 \mathrm{~s})$. In terms of decay time $\left(T_{1 / 2}\right)$, the $\mathrm{HbR}(28.2 \pm 5.9 \mathrm{~s})$ holds the longest value followed by HbT (27.0 $\left.\pm 8.6 \mathrm{~s}\right)$, $\mathrm{sO}_{2}(25.5 \pm 6.7 \mathrm{~s})$ and $\mathrm{HbO}(24.4 \pm 7.3 \mathrm{~s})$. 

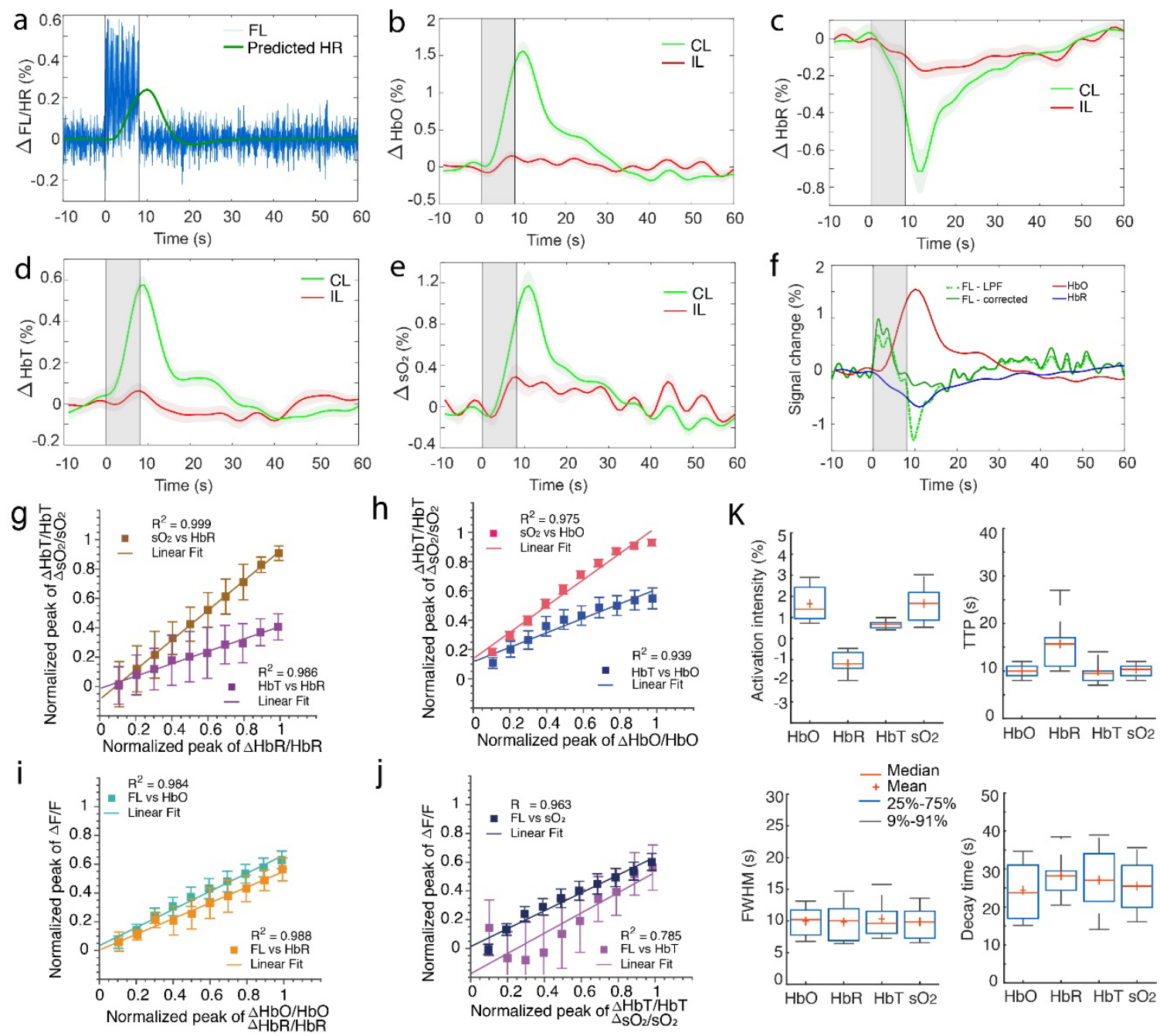

Figure 4 Multi-parametric analysis of the coupling between simultaneous fluorescence and fOA readings. a Averaged fluorescence activation curve after applying a bandpass filter $(3-8 \mathrm{~Hz})$ and the predicted hemodynamic response (HR) calculated via convolution with the HRF. b-e Averaged activation curves of $\mathrm{HbO}, \mathrm{HbR}, \mathrm{HbT}$ and $\mathrm{sO}_{2}$ across all the stimulation trials. Shaded regions show standard error of mean (SEM) across $n=6$ mice in all the trials. f Fluorescence signal compensated for hemoglobin absorption variations by considering the fractional changes of $\mathrm{HbO}$ and $\mathrm{HbR}$ from the contralateral side. g-j Coupling between $\mathrm{HbO}, \mathrm{HbR}, \mathrm{HbT}, \mathrm{sO}_{2}$ and lowpass filtered fluorescence signal from simultaneously measured single activation traces. $\mathbf{k}$ Statistics of the activation intensity, TTP, FWHM and decay time $\left(T_{1 / 2}\right)$ across all the trials from 6 mice. Note that all values are statistically significant compared to their ipsilateral counterparts ( $p$-value $<0.05$, two-tailed paired t-test). CL: contralateral, IL: ipsilateral.

\section{Discussion}

Major efforts are underway to devise new neuroimaging methods enabling scalable recording of neural activity and the associated hemodynamic responses. Both calcium and hemodynamic changes can be captured with sequential (non-concurrent) multi-modal strategies combining different contrast mechanisms. However, the mammalian brain's complexity and its highly dynamic physiological status challenge the establishment of interpretable links across separately acquired multi-modal readings often covering different spatio-temporal scales. In this work, calcium signal and hemodynamic responses were imaged concurrently and a tailored functional data processing pipeline was developed to facilitate the 
comparison of calcium and hemodynamic responses. Significant correlation was revealed between the GCaMP fluorescence signal and the associated increases in the $\mathrm{HbO}, \mathrm{HbT}, \mathrm{sO}_{2}$ and decrease in the $\mathrm{HbR}$ signals. In previous neuroimaging studies with $\mathrm{FMRI}$, it was determined that $\mathrm{HbR}$ changes are the chief contributors to the BOLD signal. Interestingly, this signal is affected by changes in blood flow, blood volume, and metabolic rate of oxygen, which makes the BOLD signal relatively ambiguous to interpret. In contrast, we were able here to retrieve signal changes from individual hemodynamic components simultaneously. The cross-correlation analysis (Figs. $\mathbf{4 g}, \mathbf{h}$ ) indicates that both $\Delta \mathrm{HbO}$ and $\Delta \mathrm{HbR}$ have comparable contribution to the local change in $\mathrm{HbT}$ and $\mathrm{sO}_{2}$ while $\Delta \mathrm{HbO}$ has slightly higher contribution to $\triangle \mathrm{HbT}$. The coupling between calcium signal and hemodynamics is further corroborated by crosscorrelation between $\Delta \mathrm{HbO} / \Delta \mathrm{HbR}$ and the hemodynamic changes $\Delta \mathrm{Hb}$ predicted by analyzing the GCaMP signal convolved with the HRF. These findings complement previous observations on event-evoked brain activation mapping and related hemodynamic readings based on wide-field optical mapping and $\mathrm{fMRI}^{2,5,14-17}$. The hybrid FLOT approach expands the capabilities of previously reported multi-modal methods to provide more comprehensive functional information on stimulus-evoked neural activation in the murine brain.

fOA is particularly advantageous for characterizing hemodynamic responses in the brain as it can map multiple hemodynamic parameters in 3D with high spatial resolution and ultrafast (real-time) volume rate. Recently it has been shown to be sensitive to fast stimulus-evoked calcium responses mediated by spectral absorption changes in GCaMP proteins ${ }^{18,19}$. However, accurate spectral unmixing of GCaMPassociated changes from the strong background absorption by hemoglobin remains challenging with fOA, especially when employing calcium-sensitive molecules with peak absorption in the visible spectral range. We have considered the possible confound where spectral unmixing of $\mathrm{HbO}$ and $\mathrm{HbR}$ with a linear model leads to quantitative errors in the $\mathrm{sO}_{2}$ and $\mathrm{HbT}$ readings due to the wavelength-dependent light attenuation in the tissue ${ }^{18,19}$. It has previously been shown that accurate estimation of oxygenation can be obtained by selecting a set of optical wavelengths that (1) minimize spectral coloring effects, (2) avoid ill-conditioning in the inverse problem, and (3) provide a sufficiently high SNR ${ }^{21}$. The wavelength combination in this work was carefully chosen to ensure optimal spectral unmixing of the fOA images. Specifically, we avoided the visible spectral range of $500-600 \mathrm{~nm}$, where significant spectral coloring associated to the strongly wavelength-dependent blood absorption is produced. Furthermore, one of the excitation wavelengths was selected at the isosbestic point of hemoglobin $(800 \mathrm{~nm})$, another wavelength at the fingerprint spectral point of $\mathrm{HbR}(755 \mathrm{~nm})$ and the remaining three wavelengths of 700,730 and $850 \mathrm{~nm}$ exhibiting large absorption difference between $\mathrm{HbR}$ and $\mathrm{HbO}$. This ensures a small condition number in the molar absorption coefficient matrix. NIR wavelengths are known to more efficiently penetrate biological tissues, thus providing a good balance between penetration depth and SNR for deep brain imaging. Note however, that fOA generally provides inferior penetration depth as compared to MRI or positron emission tomography, while the high resolution of the method can be further compromised by the skull-induced ultrasound distortions ${ }^{22}$.

Accurate estimation of the HRF shape is essential to preventing both false positives and false negatives that may result from a mismatch between the predicted and measured signals. Considering that no specific HRF model currently exists for the different hemodynamic readings provided by fOA, we employed a modified SPM canonical HRF with a TTP of $2.3 \mathrm{~s}$ and FWHM of $1.9 \mathrm{~s}$ to represent the rodent hemodynamic response ${ }^{23,24}$. This simplified model was shown to effectively infer active voxels in the mouse brain. Note, however, that the shape of the HRF has been shown to be affected by the particular selection of tasks and brain regions, thus limiting its general applicability. Also, the hemodynamic 
response to a brief stimulus in the rodent brain is species-dependent and can significantly change during development ${ }^{17}$. Ideally, the HRF parameters should be directly interpretable in terms of changes in neuronal activity and should be estimated so that statistical power is maximized. However, substantial differences among models in terms of power, bias and parameter mismatches have been reported in fMRI studies ${ }^{25}$, which are similarly expected to affect the fOA measurements. Hence, more sophisticated HRF models optimally fitting the different hemodynamic components are expected to bring more flexibility in estimating the functional parameters, which will be addressed in future work.

In summary, the unique capabilities of FLOT for simultaneous imaging of fast calcium signals associated to neural activity and the induced hemodynamic responses across the entire mouse cortex noninvasively can reveal new insights into the basic mechanisms and extent of stimulus-evoked responses. fOA is a versatile tool to resolve multiple hemodynamic parameters in real-time, thus providing additional information that may enhance or complement existing methods of studying the neurovascular and neurometabolic coupling mechanisms. Epifluorescence has high sensitivity and spatio-temporal resolution when it comes to tracking calcium activity in the cortical regions, which served to corroborate and complement the fOA observations. The fOA readings were in turn used to decouple the calcium responses from the slowly varying hemodynamic variations in the fluorescence recordings. The proposed approach does not require cranial windowing or any other invasive surgical procedures. It can also be used in a fully non-invasive manner in mice with non-pigmented scalps. All in all, FLOT is a powerful new brain imaging tool that can be broadly applied for investigations into neurovascular coupling, cerebrovascular and neurodegenerative conditions as well as monitoring of therapies.

\section{Methods}

\section{Hybrid epifluorescence and optoacoustic tomography (FLOT) system}

Hybrid epifluorescence-optoacoustic imaging was achieved with a system that integrates a spherical matrix array transducer (Imasonic SaS, Voray, France) for volumetric fOA imaging with a custom-made fiberscope (Zibra Corporation, Westport, USA) for co-registered optical (epifluorescence) imaging (Fig. 1a). The transducer array consists of 512 piezocomposite elements arranged on a hemispherical surface with a $150^{\circ}$ angular coverage ( $1.48 \pi$ solid angle). Individual elements have $7 \mathrm{MHz}$ central frequency and $>80 \%$ detection bandwidth. The array features a central $8 \mathrm{~mm}$ diameter aperture and three additional $4 \mathrm{~mm}$ diameter apertures located at $45^{\circ}$ elevation angle and equally spaced in the azimuthal direction. The fiberscope consists of a $1.4 \mathrm{~mm}$ diameter optic image guide consisting of 100,000 fibers collecting the fluorescent responses and an illumination bundle composed of 19 fibers having $600 \mu \mathrm{m}$ core diameter and 0.4 numerical aperture (NA) for optical excitation (Zibra Corporation, Westport, USA). Epifluorescence imaging was performed by inserting the tip of the fiberscope into the $8 \mathrm{~mm}$ aperture of the matrix array. At the output end of the image guide, two emission filters (FL514.5-10, Thorlabs, USA) were cascaded to isolate the fluorescence emission from GCaMP, which was subsequently captured with an EMCCD camera (iXon Life, Andor, UK) at $20 \mathrm{fps}$. The hybrid FLOT system provided a co-registered 12 $\mathrm{mm}$ diameter FOV with $40 \mu \mathrm{m}$ lateral resolution for fluorescence and nearly isotropic 3D spatial resolution down to $113 \mu \mathrm{m}$ for fOA imaging. Note that for characterizing the GCaMP6f impulse response with high temporal resolution in the millisecond range, the EMCCD camera having superior SNR was replaced with a high-speed CMOS camera (PCO.dimax S1, PCO, Germany) operating at $200 \mathrm{fps}$. Fluorescence excitation was provided with a continuous wave $488 \mathrm{~nm}$ laser (Sapphire LPX 488-500, Coherent, USA). On the other hand, a custom-made fiber bundle (CeramOptec GmbH, Germany) was 
used to guide a short-pulsed ( $<10 \mathrm{~ns}$ ) beam generated with an optical parametric oscillator (OPO) laser (Spit-Light, Innolas Laser $\mathrm{GmbH}$, Germany) through the three lateral apertures of the array. The laser wavelength was rapidly swept between five wavelengths $(700 \mathrm{~nm}, 730 \mathrm{~nm}, 755 \mathrm{~nm}, 800 \mathrm{~nm}$, and $850 \mathrm{~nm}$ ) on a per-pulse basis at $100 \mathrm{~Hz}$ pulse repetition frequency (PRF). The pulse energy was $\sim 11 \mathrm{~mJ}$ at the output of the illumination fiber bundle. The generated signals were acquired with a custom-made data acquisition system (DAQ, Falkenstein Mikrosysteme $\mathrm{GmbH}$, Germany) at 40 Megasamples per second (Msps) and recorded raw data transmitted to a PC via Ethernet. Synchronization of the excitation light pulses, the fluorescence and fOA data acquisition, and the electrical paw stimulation was achieved with an external trigger device (Pulse Pal V2, Sanworks, USA).

\section{Animal models}

Female GCaMP6f mice (C57BL/6J-Tg(Thy1-GCaMP6f) GP5.17Dkim/J, the Jackson Laboratory, USA, 6 to 11 week-old, $n=6$ ) were employed in this study. Animals were housed in individually ventilated, temperature-controlled cages under a 12-hour dark/light cycle. Pelleted food (3437PXL15, CARGILL) and water were provided ad-libitum. All experiments were performed in accordance with the Swiss Federal Act on Animal Protection and were approved by the Cantonal Veterinary Office Zurich.

\section{In vivo imaging}

Mice were anesthetized for the in vivo imaging experiments. Anesthesia was inducted with intraperitoneal injection of ketamine $(100 \mathrm{mg} / \mathrm{kg}$ body weight, Pfizer) and xylazine $(10 \mathrm{mg} / \mathrm{kg}$ body weight, Bayer) cocktail. The bolus injections were separated into two injections with 5 min gap to prevent cardiovascular complications. Maintenance injection was administrated every 45 min with a mixture of ketamine (100 mg/kg body weight) and xylazine $(2.5 \mathrm{mg} / \mathrm{kg}$ body weight). Both the scalp and skull of the mice were kept intact $(n=4)$ on the premise that no pigment in the scalp were presented while the scalp was removed and skull was kept intact for mice with scalp pigmentation $(n=2)$. Scalp removal was performed after injection of Buprenorphine $(0.1 \mathrm{mg} / \mathrm{kg}$ body weight, Temegesic, Indivior, Switzerland) together with hemostatic sponges (Gelfoam ${ }^{\circledR}$, Pfizer Pharmaceutical) to minimize bleeding. Imaging was performed by placing each mouse onto a 3D translation stage in a prone position that facilitated optimal positioning of the region of interest in the brain in the center of the FOV of both modalities. Ultrasound gel was applied on the mouse head to ensure optimal acoustic signal coupling. The mouse head was immobilized using a custom stereotactic frame (Narishige International Limited, London, United Kingdom). During the experiment, an oxygen/air mixture (0.1/0.4 L. $\left.\mathrm{min}^{-1}\right)$ was provided through a breathing mask. Peripheral blood oxygen saturation, heart rate and body temperature were continuously monitored (PhysioSuite, Kent Scientific) during data acquisition. The body temperature was kept around $37^{\circ} \mathrm{C}$ with a feedback-controlled heating pad.

A BOLD fMRI comparable stimulation paradigm in terms of stimulation duration and intensity was adopted in this study ${ }^{15}$. Unipolar rectangular electric pulses of $5 \mathrm{~ms}$ duration and $1.0 \mathrm{~mA}$ intensity were applied to the left hindpaw at $4 \mathrm{~Hz}$ stimulus frequency, $8 \mathrm{~s}$ onset time, and $82 \mathrm{~s}$ burst intervals (Fig. 1b). The electric signals were generated using a stimulus isolator device (Model A365R, World Precision Instruments, USA) fed by an external trigger (Pulse Pal V2, Sanworks, USA). For each sequence, the total duration of the stimulation pulse train was $440 \mathrm{~s}$ with the individual stimuli synchronized with the excitation light and data acquisition system. The sequence was repeated 2-5 times per animal. After the experiments, the animals with scalp removed were euthanized while still under anesthesia, whereas other mice with intact scalp emerged from the anesthesia and fully recovered. 


\section{Custom data analysis pipeline for both fluorescence and fOA}

A data analysis pipeline inspired by SPM12 $2^{26,27}$ was developed for both fluorescence and fOA data analysis (Fig. 1e). To this end, a custom script was developed with MATLAB (2019b, MathWorks, USA). A general linear model (GLM) was applied to a voxel-by-voxel based statistical analysis. fOA volumetric data and fluorescence images were pre-processed before being fed into the model. Specifically, fOA raw signal matrices were first separated according to the corresponding excitation wavelengths and the signals bandpass filtered between $0.1-8 \mathrm{MHz}$. The images were reconstructed separately for each wavelength using a filtered back-projection algorithm ${ }^{28}\left(100 \times 100 \times 100 \mu \mathrm{m}^{3}\right.$ voxel size, $\left.8 \times 8 \times 4 \mathrm{~mm}^{3} \mathrm{FOV}\right)$. Generally, the fOA signal at an arbitrary point $r$ within the imaged tissue volume is mainly resulting from the light absorption by $\mathrm{HbO}$ and $\mathrm{HbR}$, which can be approximated $\mathrm{as}^{29}$

$$
\operatorname{sig}_{f O A T}(\boldsymbol{r}, \lambda) \approx \Gamma(\boldsymbol{r}) \phi(\boldsymbol{r}, \lambda)\left[\varepsilon_{H b O}(\lambda) C_{H b O}(\boldsymbol{r})+\varepsilon_{H b R}(\lambda) C_{H b R}(\boldsymbol{r})\right]
$$

where $\Gamma$ is the Grueneisen parameter, $\lambda$ is the wavelength of the illumination laser beam, $\phi$ is the light fluent within the tissue and $\varepsilon$ and $C$ are the molar extinction coefficients and concentrations of each absorbing substance (molecule), respectively. fOA signals were firstly normalized to the laser pulse energy at each wavelength. To reduce the spectral coloring effects ${ }^{21}$, i.e., the spatially and wavelength dependent light fluence distribution within the tissue, an exponential light attenuation model was applied to compensate for the depth-dependent signal decay. The effective attenuation coefficient ( $\mu_{\text {eff }}$ ) was calculated as ${ }^{30}$

$\mu_{e f f}=\left[3 \mu_{a}\left(\mu_{a}+\mu_{s}^{\prime}\right)\right]^{1 / 2}$,

where $\mu_{\mathrm{a}}$ is the absorption coefficient and was set to 0.1 , assuming homogenous light absorption in the mouse brain at all the 5 wavelengths. $\mu_{s}^{\prime}=a\left(\frac{\lambda}{500(n m)}\right)^{-b}$ is the reduced scattering coefficient whereas $a=24.2 \mathrm{~cm}^{-1}$ and $b=1.611$ were assumed based on the averaged scattering properties of the brain tissue 30 .

After light fluence correction, the concentrations of $\mathrm{HbO}$ and $\mathrm{HbR}$ for a spatially independent $\Gamma$ were estimated via linear spectral unmixing as

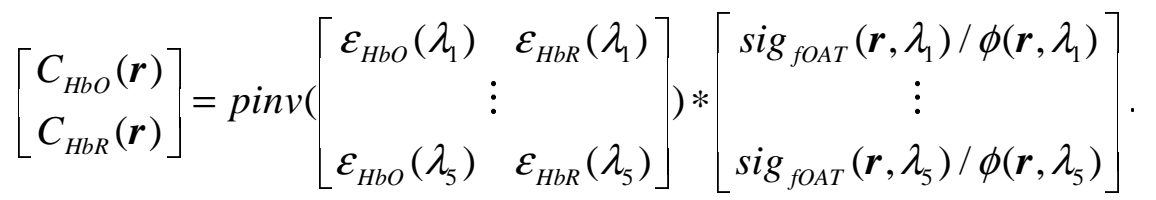

Subsequently, $\mathrm{HbT}$ and $\mathrm{sO}_{2}$ were calculated from the concentrations of $\mathrm{HbO}$ and $\mathrm{HbR}$ as

$$
\begin{aligned}
& C_{H b T}(\boldsymbol{r})=C_{H b O}(\boldsymbol{r})+C_{H b R}(\boldsymbol{r}), \\
& s O_{2}(r)=\frac{C_{H b O}(\boldsymbol{r})}{C_{H b O}(\boldsymbol{r})+C_{H b R}(\boldsymbol{r})} .
\end{aligned}
$$

For fluorescence image pre-processing, the image stack was rearranged to form a 4D volume with the third dimension equal to 1 to make it compatible to the overall processing pipeline. Motion estimation 
and correction was performed by using SPM12 realign module before the processed data was fed to the model for statistical analysis. The custom stereotactic frame helped mitigating the motion artifacts. The relative center of mass displacement in each trial typically remained below 0.2 voxel, thus ensuring negligible motion-induced errors (false activations). Spatial smoothing (Gaussian kernel FWHM=0.3 mm) was further applied to reduce random fluctuations and reconstruction-related image artifacts. A highpass filter of $1 / 135 \mathrm{~Hz}$ was then used to detrend the fOA data. For fluorescence data, a lowpass filter with $0.5 \mathrm{~Hz}$ cutoff frequency was applied to retrieve the slow hemodynamic response encoded in the fluorescence signal and a bandpass filter $(3-8 \mathrm{~Hz})$ was applied to extract the fast calcium transients.

In the GLM, the regressor was obtained by convolving the stimulation paradigm (Fig. 1b) with either the measured GCaMP CRF (for fluorescence data) or a modified SPM canonical HRF (for fOA data) having a TTP of $2.3 \mathrm{~s}$ and FWHM of $1.9 \mathrm{~s}$ to represent the rodent hemodynamic response ${ }^{23,24}$. The regressor together with a constant vector formed the design matrix. The GLM is expressed in the matrix format via $^{31}$

$Y=X \beta+\varepsilon$,

where $Y$ is the column vector of observations representing a signal voxel sampled at successive time point, $\varepsilon$ is the column vector of error terms, $\beta$ is the column vector of parameters and $X$ is the design matrix. The estimated parametric map is calculated as

Betas $=\operatorname{inv}(X) * Y$,

The statistical significance of the event-evoked responses in the observations was further evaluated with a contrast vector $c=[1,0]^{\top}$. The $t$ statistic map (i.e. $t$-map) and probability levels (i.e. $p$-values) were calculated as the statistical inferences. Note that for rendering the activation map for fOA, the false discovery rate (FDR) controlling ${ }^{32}$ with $p<0.05$ was introduced to the t-map. For the fluorescence recordings, the parameteric map (Betas in Eq. (7)) was used directly to infer the activation.

The activation map and the corresponding structural image acquired at $850 \mathrm{~nm}$ excitation wavelength were overlaid to the Allen Mouse Brain Atlas $2015^{33}$. The atlas volume has $320 \times 456$ pixels in each coronal plane and consists of 528 slices with $25 \mu \mathrm{m}$ isotropic voxel size. Due to the lack of common features and utterly different intensity distribution, unbiased automated registration of fOA with MRI remains challenging ${ }^{34}$. In this work, the commonly used manual landmark based coregistration method ${ }^{35}$ was employed for the alignment in Amira (version 5.4, Thermo Fisher Scientific, USA). Firstly, the structural volumetric data of the mouse brain was reconstructed with the averaged signal matrix acquired at $850 \mathrm{~nm}$. Secondly, this structural volume and the mouse brain atlas were loaded into Amira for pre-alignment. A coarse transformation with respect to translation and rotation was tuned according to the landmarks in both datasets. Finally, the position of the structural optoacoustic volumetric data was finetuned based on cross-sections along the three planes (perspectives) so that landmarks such as the brain curvature and vessel structures matched the MRI brain atlas. The calculated transform information in the transform editor in Amira facilitated registering all the other reconstructed parameters such as $\mathrm{HbO}, \mathrm{HbR}, \mathrm{HbT}$ and $\mathrm{SO}_{2}$ from the same mouse to the brain atlas. The overlaid activation map was subsequently rendered and displayed along different planes.

The time courses of brain activation were retrieved from the raw 4D data, namely, 3D volume time series for $f O A$ and 2D image series for fluorescence data. The calculated activation map served to infer responsive ROls in both contralateral and ipsilateral hemispheres. A time window including $10 \mathrm{~s}$ pre- 
stimulation, $8 \mathrm{~s}$ onset and $50 \mathrm{~s}$ post-stimulation was selected. Baseline signals of each stimulation cycle were calculated by averaging the $10 \mathrm{~s}$ pre-stimulation time window. This was used for calculating fractional signal changes, which were averaged across different stimulation cycles. All the trials acquired at slightly different anesthesia depth and from different mice were averaged to reveal the stimulusevoked brain activation in a more robust way. Statistics on activation intensity, TTP, FWHM as well as decay time $\left(T_{1 / 2}\right)$ were further performed across all the trials. The statistical significance between the averaged values from the contralateral side and their ipsilateral counterparts were evaluated with twotailed paired t-test.

\section{Acknowledgements}

The authors acknowledge grant support from US National Institutes of Health (UF1 NS107680) and the European Research Council (ERC-2015-CoG-682379).

\section{Conflict of interest}

The authors declare no conflicts of interest.

\section{Ethics statement}

All procedures involving mice conformed to the national guidelines of the Swiss Federal Act on animal protection and were approved by the Cantonal Veterinary Office Zurich.

\section{Author contributions}

ZC and DR conceived the concept and devised the study. ZC and QZ performed the animal experiments. ZC, IG performed image reconstructions and data analysis. XLDB, RN and MR helped with the experiments and provided helpful discussions. SS and DR supervised the work. All authors contributed to writing and revising the manuscript.

\section{References}

1 Glover G H, Overview of functional magnetic resonance imaging. Neurosurg Clin N Am 2011; 22: 133.

2 Blockley N P, Griffeth V E M, Simon A B, Buxton R B, A review of calibrated blood oxygenation leveldependent (bold) methods for the measurement of task-induced changes in brain oxygen metabolism. NMR Biomed 2013; 26: 987-1003.

3 Aydin A, Haselden W D, Goulam Houssen Y, Pouzat C, Rungta R L et al., Transfer functions linking neural calcium to single voxel functional ultrasound signal. Nat Commun 2020; 11: 2954.

4 Sun X R, Badura A, Pacheco D A, Lynch L A, Schneider E R et al., Fast gcamps for improved tracking of neuronal activity. Nat Commun 2013; 4: 2170.

5 Ma Y, Shaik M A, Kozberg M G, Kim S H, Portes J P et al., Resting-state hemodynamics are spatiotemporally coupled to synchronized and symmetric neural activity in excitatory neurons. Proceedings of the National Academy of Sciences 2016; 113: E8463.

6 Deán-Ben X L, Gottschalk S, Mc Larney B, Shoham S, Razansky D, Advanced optoacoustic methods for multiscale imaging of in vivo dynamics. Chem Soc Rev 2017; 46: 2158-2198.

7 Mc Larney B, Hutter M A, Degtyaruk O, Deán-Ben X L, Razansky D, Monitoring of stimulus evoked murine somatosensory cortex hemodynamic activity with volumetric multi-spectral optoacoustic tomography. Front Neurosci-Switz 2020; 14: 536.

8 Deán-Ben X L, Gottschalk S, Mc Larney B, Shoham S, Razansky D, Advanced optoacoustic methods for multiscale imaging of in vivo dynamics. Chem Soc Rev 2017; 46: 2158-2198.

$9 \quad$ Ovsepian S V, Jiang Y, Sardella T C P, Malekzadeh-Najafabadi J, Burton N C et al., Visualizing cortical response to optogenetic stimulation and sensory inputs using multispectral handheld optoacoustic imaging. Photoacoustics 2020; 17: 100153. 
10 Chen Q, Cichon J, Wang W, Qiu L, Lee S J et al., Imaging neural activity using thy1-gcamp transgenic mice. Neuron 2012; 76: 297-308.

11 Chen Z, Deán-Ben X L, Liu N, Gujrati V, Gottschalk S et al., Concurrent fluorescence and volumetric optoacoustic tomography of nanoagent perfusion and bio-distribution in solid tumors. Biomed Opt Express 2019; 10: 5093-5102.

12 Chen Z, Dean-Ben X L, Gottschalk S, Razansky D, Hybrid system for in vivo epifluorescence and 4d optoacoustic imaging. Opt Lett 2017; 42: 4577-4580.

13 Bakker R, Tiesinga P, Kötter R, The scalable brain atlas: instant web-based access to public brain atlases and related content. Neuroinformatics 2015; 13: 353-366.

14 Ma Y, Shaik M A, Kim S H, Kozberg M G, Thibodeaux D N et al., Wide-field optical mapping of neural activity and brain haemodynamics: considerations and novel approaches. Philosophical transactions of the Royal Society of London. Series B, Biological sciences 2016; 371: 20150360.

15 Shim H J, Jung W B, Schlegel F, Lee J, Kim S et al., Mouse fmri under ketamine and xylazine anesthesia: robust contralateral somatosensory cortex activation in response to forepaw stimulation. Neuroimage 2018; 177: 3044.

16 Lebhardt P, Hohenberg C C, Weber-Fahr W, Kelsch W, Sartorius A, Optogenetic fmri in the mouse hippocampus: hemodynamic response to brief glutamatergic stimuli. J Cereb Blood Flow Metab 2016; 36: 629-638.

17 Arichi T, Fagiolo G, Varela M, Melendez-Calderon A, Allievi A et al., Development of bold signal hemodynamic responses in the human brain. Neuroimage 2012; 63: 663-673.

18 Gottschalk S, Degtyaruk O, Mc Larney B, Rebling J, Hutter M A et al., Rapid volumetric optoacoustic imaging of neural dynamics across the mouse brain. Nat Biomed Eng 2019; 3: 392-401.

19 Deán-Ben X L, Sela G, Lauri A, Kneipp M, Ntziachristos V et al., Functional optoacoustic neurotomography for scalable whole-brain monitoring of calcium indicators. Light: Science \& Applications 2016; 5: e16201.

${ }_{20}$ Wang M, Wu C, Sinefeld D, Li B, Xia F et al., Comparing the effective attenuation lengths for long wavelength in vivo imaging of the mouse brain. Biomed Opt Express 2018; 9: 3534-3543.

21 Roman H, Lu A, Paul C B, Benjamin T C, Estimating blood oxygenation from photoacoustic images: can a simple linear spectroscopic inversion ever work? J Biomed Opt 2019; 24: 1-13.

22 Estrada H, Gottschalk S, Reiss M, Neuschmelting V, Goldbrunner R et al., Observation of guided acoustic waves in a human skull. Ultrasound in Medicine \& Biology 2018; 44: 2388-2392.

23 Silva A C, Koretsky A P, Duyn J H, Functional mri impulse response for bold and cbv contrast in rat somatosensory cortex. Magn Reson Med 2007; 57: 1110-1118.

24 Berwick J, Johnston D, Jones M, Martindale J, Redgrave P et al., Neurovascular coupling investigated with two-dimensional optical imaging spectroscopy in rat whisker barrel cortex. Eur J Neurosci 2005; 22: 1655-1666.

25 Lindquist M A, Meng Loh J, Atlas L Y, Wager T D, Modeling the hemodynamic response function in fmri: efficiency, bias and mis-modeling. Neuroimage 2009; 45: S187-S198.

26 https://www.fil.ion.ucl.ac.uk/spm/software/spm12/

27 Flandin G, Novak M J U, in fMRI: Basics and Clinical Applications, edited by Stephan Ulmer and Olav Jansen (Springer Berlin Heidelberg, Berlin, Heidelberg, 2013), pp. 51-76.

28 Wang L V, Xu M, Universal back-projection algorithm for photoacoustic computed tomography. Phys Rev E 2005; 71: 16706.

29 Deán-Ben X L, Bay E, Razansky D, Functional optoacoustic imaging of moving objects using microseconddelay acquisition of multispectral three-dimensional tomographic data. Sci Rep-Uk 2014; 4: 5878.

$30 \quad$ Jacques S L, Optical properties of biological tissues: a review. Phys Med Biol 2013; 58: R37-R61.

31 Friston K J, Holmes A P, Worsley K J, Poline J P, Frith C D et al., Statistical parametric maps in functional imaging: a general linear approach. Hum Brain Mapp 1994; 2: 189-210.

32 Pendse G, Borsook D, Becerra L, Enhanced false discovery rate using gaussian mixture models for thresholding fmri statistical maps. Neuroimage 2009; 47: 231-261.

33 Lein E S, Hawrylycz M J, Ao N, Ayres M, Bensinger A et al., Genome-wide atlas of gene expression in the adult mouse brain. Nature 2007; 445: 168-176.

34 Ren W, Skulason H, Schlegel F, Rudin M, Klohs J et al., Automated registration of magnetic resonance imaging and optoacoustic tomography data for experimental studies. Neurophotonics 2019; 6: 25001.

35 Attia A B E, Ho C J H, Chandrasekharan P, Balasundaram G, Tay H C et al., Multispectral optoacoustic and mri coregistration for molecular imaging of orthotopic model of human glioblastoma. J Biophotonics 2016; 9: 701-708. 
bioRxiv preprint doi: https://doi.org/10.1101/2021.11.08.467725; this version posted November 10, 2021. The copyright holder for this preprint (which was not certified by peer review) is the author/funder, who has granted bioRxiv a license to display the preprint in perpetuity. It is made available under aCC-BY-NC-ND 4.0 International license. 